\title{
Research Paper \\ Effectiveness of Integrated and Comprehensive Geriatric Care Program of Minis- try of Health on Physical and Mental Health of Older Adults in Golestan Province
}

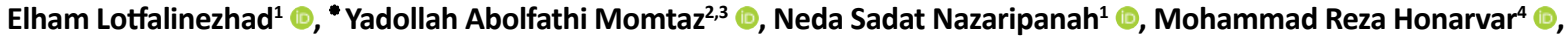 \\ Fatemeh Arab Ameri ${ }^{5} \odot$, Mahin Sadat Azimi ${ }^{6}$ (ㅇ
}

1. Department of Health Education and Promotion, Faculty of Health, Tabriz University of Medical Sciences, Tabriz, Iran.

2. Iranian Research Center on Aging, Faculty of Health, University of Social Welfare and Rehabilitation Sciences, Tehran, Iran.

3. Malaysian Research Institute on Ageing (MyAgeing), Universiti Putra Malaysia, Selangor Darul Ehsan, Malaysia.

4. Health Management and Social Development Research Center, Golestan University of Medical Sciences, Gorgan, Iran.

5. Middle-Aged and Elderly Health Group, Golestan Health Center, Gorgan, Iran.

6. Department of Statistics and Health Economics, The Ministry of Health, Tehran, Iran.

\begin{tabular}{l|l}
$\begin{array}{c}\text { Use vour devic to scan } \\
\text { and read the article online }\end{array}$ & $\begin{array}{l}\text { Cltation: Lotfalinezhad E, Abolfathi Momtaz Y, Nazaripanah NS, Honarvar MR, Arab Ameri F, Azimi MS. [Effectiveness of Inte- } \\
\text { grated and Comprehensive Geriatric Care Program of Ministry of Health on Physical and Mental Health of Older Adults in Golestan }\end{array}$ \\
Province, 2019 (Persian)]. Iranian Journal of Ageing. 2021; 16(3):438-451. http://dx.doi.org/10.32598/sija.2020.16.3.2190.2
\end{tabular}

\section{(1) (3)}

Accepted: 22 Feb 2020

Available Online: 01 Oct 2021

Keywords:

Comprehensive health

care, Geriatric

assessment, Mental

health, Aging, Goles-

tan province

\section{A BSTRACT}

Objectives In order to maintain and improve older adults' health, the integrated and comprehensive geriatric care program has been developed and run by ministry of health. The present study was conducted to evaluate the effectiveness of this program on physical and mental health of older adults in Golestan province in 2019.

Methods \& Materials The present retrospective cohort study employed a multistage clustered random sampling technique to obtain 500 e-health records of older adults during 2017-2019. The data on blood pressure, depression, and BMI were extracted from e-health records. Data analysis was conducted using repeated measure anova, paired t-test and Wilcoxon tests. The SPSS statistics software v. 20 was used to perform data analysis

Results The total number of records was 500 , of which $63.4 \%$ was related to women. The mean age of participants was $69.97 \pm 7.45$. The mean level of systolic blood pressure was significantly reduced $(P<0.001)$, while the level of depression was significantly increased $(P \leq 0.05)$. However, no significant change was observed in the body mass index.

Conclusion The results of this study showed that the integrated and comprehensive geriatric program was effective for controlling blood pressure, however was not effective for preventing depression. Therefore, stakeholders should pay more attention towards establishing appropriate program to improve older adults' mental health.

\section{* Corresponding Author:}

Yadollah Abolfathi Momtaz, PhD.

Address: Iranian Research Center on Aging, Faculty of Health, University of Social Welfare and Rehabilitation Sciences, Tehran, Iran.

Tel: +98 (41) 33357582

E-mail: yabolfathi@gmail.com 


\section{Extended Abstract}

\section{Introduction}

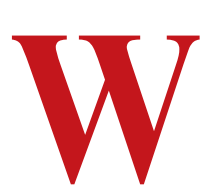

ith regard to the gradual improvements in the provision of health services, life expectancy has increased, which has resulted in an increase in the percentage of the older population. According to available statistics, older people in comparison to other age groups are examined five or seven times by doctors. More than $60 \%$ of medical expenses are spent for older people [4]. Therefore, it is proposed that there should be a fundamental change in the provision of health care for older population. In Iran, the comprehensive health care program for older adults is included. In this model, the minimum risk factors and clinical symptoms are applied to detect early disease identification, referral and appropriate treatment [7]. The purpose of this study was to investigate the effectiveness of Integrated and Comprehensive Geriatric Care Program of Ministry of Health on Physical and Mental Health of Older Adults in Golestan Province, 2019.

\section{Materials and Method}

This was a historical cohort study that was conducted in 2019. This province is located on the North-East of Iran, according to the last census in 2016, the percent of older individuals aged at 60 and over both in rural and urban regions is 7.81 .

Data collection was carried out from 25 March to 14 may 2019. Five hundred health electronic records from 130 com- prehensive rural health centers were selected by stratified multi-stage cluster random sampling method. First of all, thirteen centers were randomly selected from 130 comprehensive rural health care. Then, health electronic records that had at least two follow-up examinations about blood pressure, mental health and body mass index from 2016 to 2019 were randomly reviewed. Data analyses were conducted using IBM SPSS v. 20. The statistical tests including repeated measure ANOVA, paired t-test and Wilcoxon were used.

\section{Results}

Five-hundred E-Health Records were evaluated. The Mean \pm SD age of participants was $69.97 \pm 7.45$ which was ranged from 60 and 108 year-old. The majority of candidates were women $(n=317,63.4 \%)$. Approximately, 56 per cent of participants were housewife whereas the only 1 per cent of candidates were unemployment. Most participants had informal education $(n=370,74 \%)$. In term of marital status, 72.8 per cent of participants were married. The majority of participants were Fars.

The data related to Health indicators was not appropriate. There was contradictory between systolic and diastolic blood pressure indicators at the first and third stage, the percentage of these indicators were $94 \%$ and $21.8 \%$, respectively Table 1 . Indicates the health indicators based on blood pressure, weight, height and depression.

The lowest percent was related to depression records (1 per cent at the third stage). Repeated measure ANOVA result of systolic blood pressure revealed that there was significant differences among three stages $(\mathrm{P}<0.01, \mathrm{~F}=7.44)$,

Table 1. Health indicators based on blood pressure, weight, height and depression

\begin{tabular}{|c|c|c|c|c|}
\hline \multirow{2}{*}{ Indicator } & \multirow{2}{*}{ Condition } & \multicolumn{3}{|c|}{ No. (\%) } \\
\hline & & The First Stage & The Second Stage & The Third Stage \\
\hline \multirow{2}{*}{$\begin{array}{l}\text { Systolic blood pres- } \\
\text { sure }\end{array}$} & Registered & 470(94) & 468(93.6) & $109(21.8)$ \\
\hline & Unregistered & $30(6)$ & $32(6.4)$ & $391(78.2)$ \\
\hline \multirow{2}{*}{$\begin{array}{l}\text { Diastolic blood pres- } \\
\text { sure }\end{array}$} & Registered & 470(94) & 468(93.6) & $109(21.8)$ \\
\hline & Unregistered & $30(6)$ & $32(6.4)$ & $391(78.2)$ \\
\hline \multirow{2}{*}{ Weight } & Registered & $107(21.4)$ & $105(21)$ & $31(6.2)$ \\
\hline & Unregistered & $393(78.6)$ & $395(79)$ & 469(93.8) \\
\hline \multirow{2}{*}{ Height } & Registered & $108(21.6)$ & $110(22)$ & $31(6.2)$ \\
\hline & Unregistered & $392(78.4)$ & $390(78)$ & 469(93.8) \\
\hline \multirow{2}{*}{ Depression } & Registered & $150(30)$ & $149(29.8)$ & $5(1)$ \\
\hline & Unregistered & $350(70)$ & $351(70.2)$ & 495(99) \\
\hline
\end{tabular}


Table 2. Repeated measure ANOVA results on systolic and diastolic blood pressure

\begin{tabular}{ccccc}
\hline Variable & df & Average of Squares & F & P \\
\hline Systolic blood pressure & 2 & 1069 & 7.44 & 0.002 \\
Diastolic blood pressure & 1.65 & 114.12 & 1.7 & 0.19 \\
\hline
\end{tabular}

Table 3. The result of paired-T test on body mass index

\begin{tabular}{cccccc}
\hline Variable & $\mathbf{t}$ & df & $\mathbf{P}$ & \multicolumn{2}{c}{$\mathbf{9 5 \% \mathrm { Cl }}$} \\
\cline { 5 - 6 } $\begin{array}{c}\text { Body mass at first and second } \\
\text { stage }\end{array}$ & -0.12 & 103 & 0.9 & Lower Bound & Upper Bound \\
\hline
\end{tabular}

whereas in terms of diastolic blood pressure there was not any significant difference among three stages $(\mathrm{P}>0.05, \mathrm{~F}=$ 1.7) Table 2. Demonstrates the results of Repeated measure ANOVA on systolic and diastolic blood pressure.

The paired-T test highlighted that no significant differences was identified between body mass index at the first and the second phase $(\mathrm{P}>0.05, \mathrm{t}=-0.12)$. Figure 1 and 2 present the estimated marginal means of systolic and diastolic blood pressure at three phases, and there was a clear trend of decreasing systolic blood pressure (Figure 1).

The paired-T test highlighted that no significant differences was identified between body mass index at the first and the second phase $(\mathrm{P}>0.05, \mathrm{t}=-0.12)$ Table 3 . Reveals the result of paired-T test on body mass index. The Mean \pm SD related to depression indicator (DGS11) for the first and the second phase were $1.56 \pm 2.12$ and $2.05 \pm 2.52$, respectively. Wilcoxon

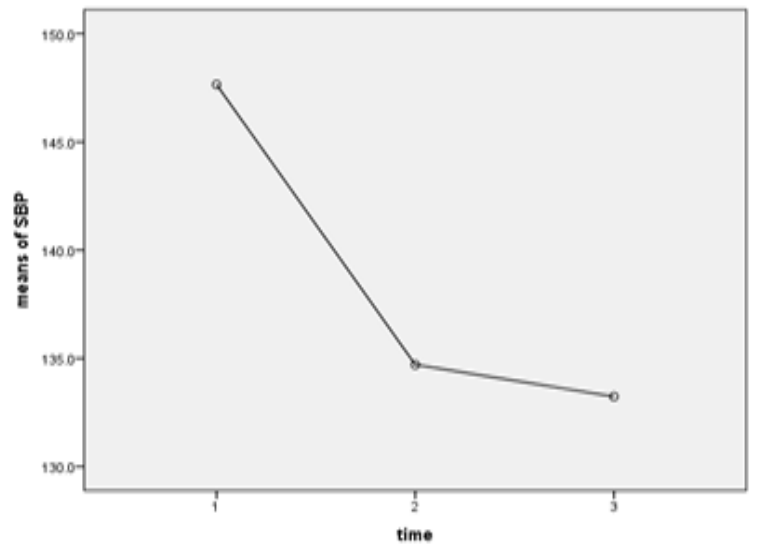

SAL M A N D

Figure 1. The marginal means of systolic blood pressure at three phases test did confirm a significant differences between the mean score of depression at the first and second phases $(\mathrm{P}<0.05)$.

\section{Discussion and conclusion}

Information related to three stages of health care followup were not complete. For instance, the researcher did not access to data related to the third stage of care assessment on depression and body mass index. The results of the current study indicated that the implementation of comprehensive health care program in golestan province was able to successfully screen and assess older adults on blood pressure but has not been successful in screening metal health (depression) and body mass index.

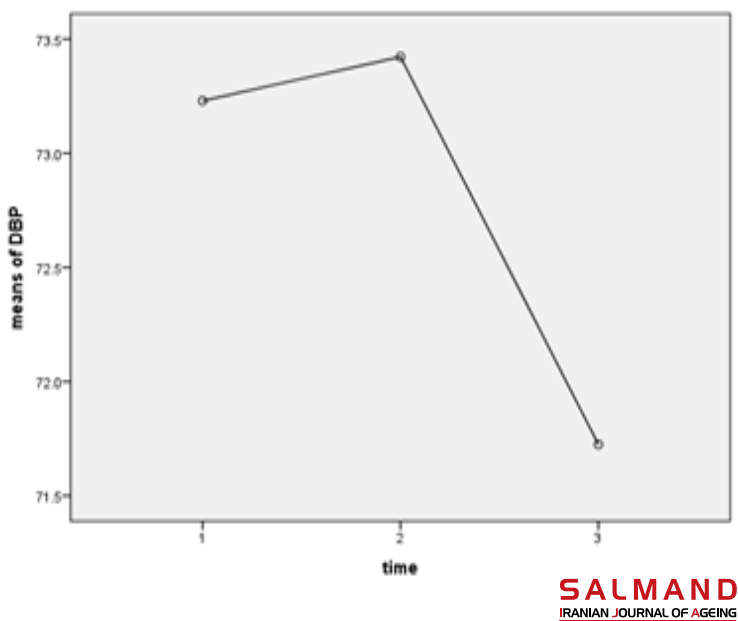

Figure 2. The marginal means of diastolic blood pressure at three phases 


\section{Ethical Considerations}

Compliance with ethical guidelines

The study was approved by the Research Committee of University of Social Health and Rehabilitation Sciences (Code: IR.USWR.REC.1397.109).

\section{Funding}

This study was supported by Research Center of University of Social Health and Rehabilitation Sciences.

\section{Authors' contributions}

All author contributed equally in preparing this article

\section{Conflicts of interest}

The authors declare no conflict of interest

\section{Acknowledgements}

We would like to express our special thanks of gratitude to Health Care Staffs of Golestan Province who cooperated us in this project. 
This Page Intentionally Left Blank 


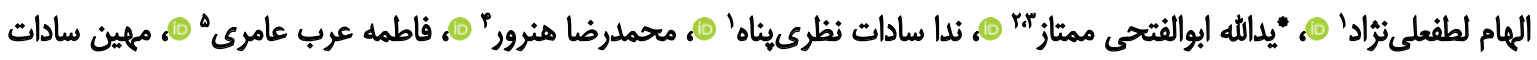

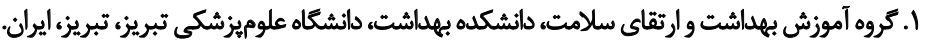

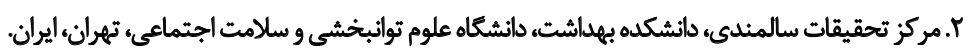

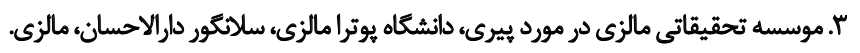

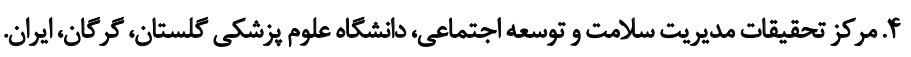

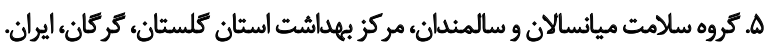
و. كروه آمار و اقتصاد سلامت، وزارت بهيناشت، تهران، ايران.

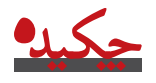

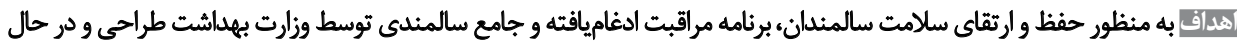

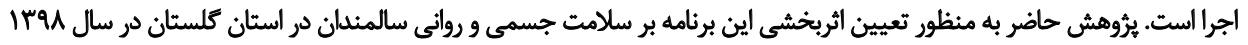

$$
\text { انجام شد. }
$$

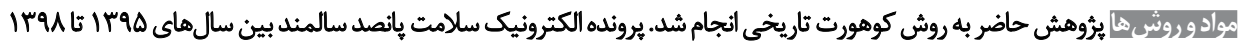

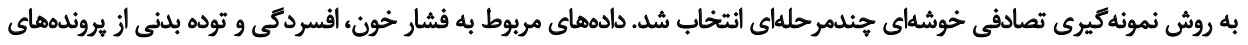

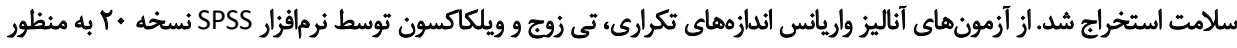
تجزيه و تحليل دادها استفاده شد.

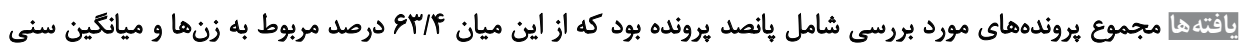

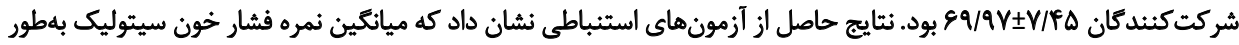

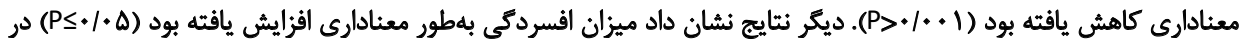

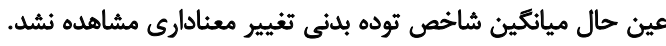

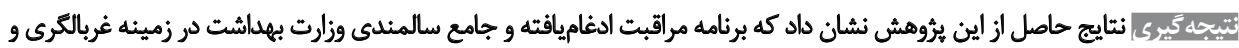

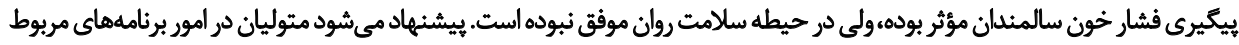

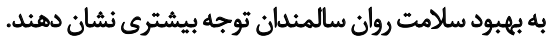

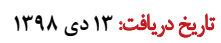

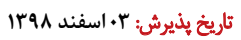 \\ تاريخ انتشار: 9. مهر ب. ب.
}

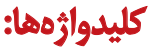

مراقبث هاى جامع

سلامت الرزياتي هاي جامع

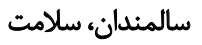

روان، سالمندى، استئان

كملستان

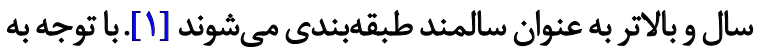

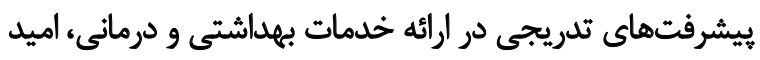

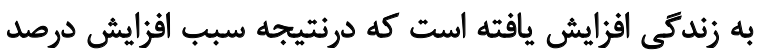

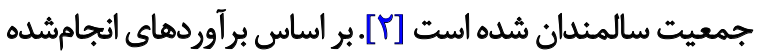

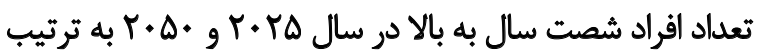
به r/ ا و r ميليارد نفر خواهد رسيد.

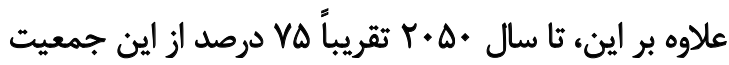

سالمند شدن جمعيت، فرايندى طبيعى است. با وجود اين،

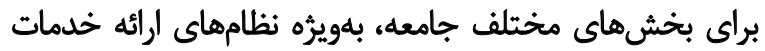

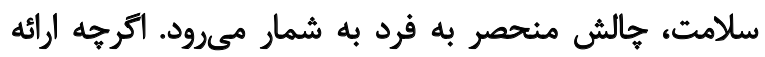

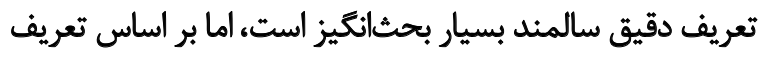

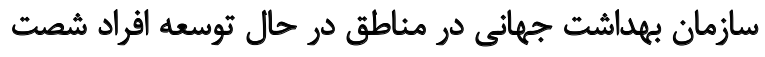

\section{-}


يك يا جند بيمارى وجود داشته باشد، سالمند از مراقبتهاي

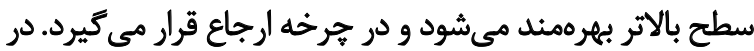

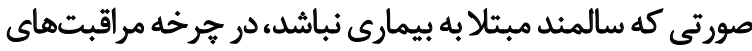

دورماى قرار مي گيرد [V]

با توجه به اينكه استان كلستان يكى از اولين استانهايى بود

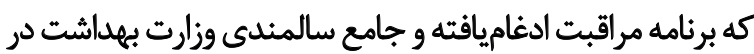

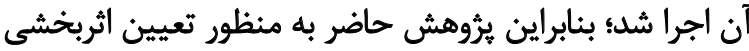

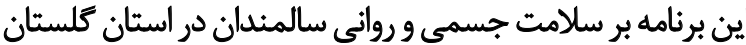

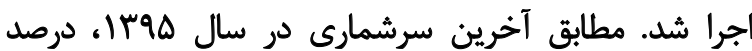

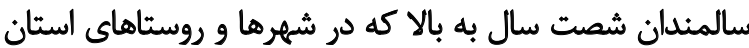

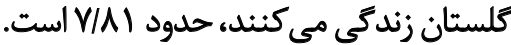
اجراي راهنماي مراقبتهاي ادغاميافته و جامع سالمندى بر بر

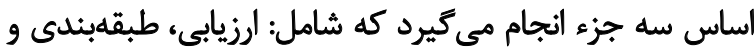

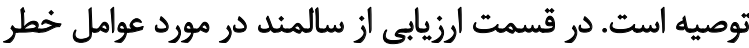

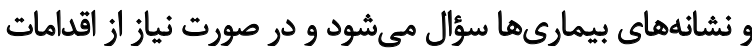

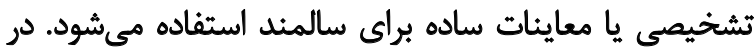

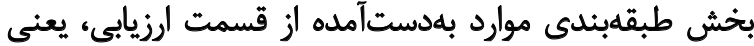

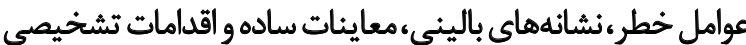

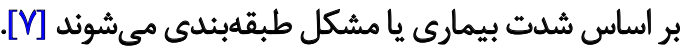

در صورتى كه سالمند نشانه و علامت بيمارى باى دارد كه خطر

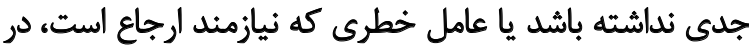

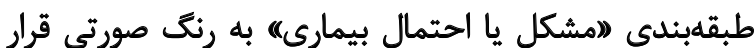

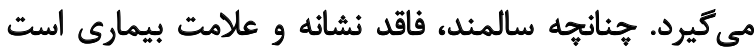

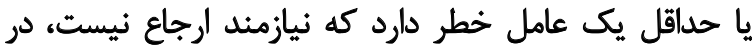

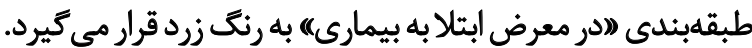

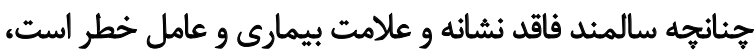
در طبقهبندى "افاقد مشكل يا بيمارى احتمالىه به به رنك سبز قرار مى كيرد [V]

در ستون مربوط به توصيهها اقدامات درمانى ساده از قبيل

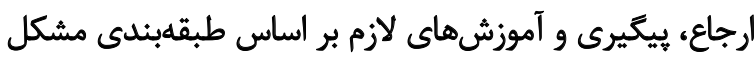

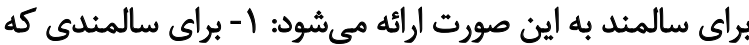

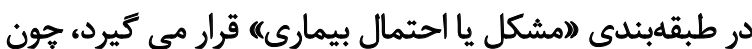

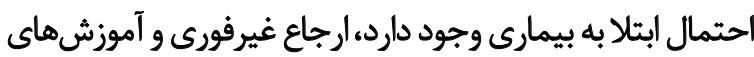

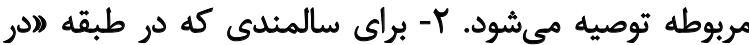

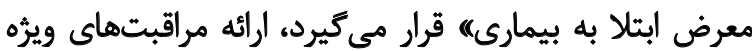

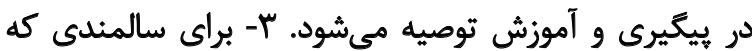

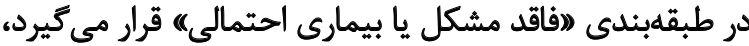

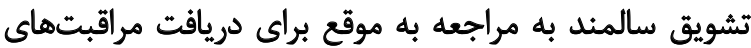

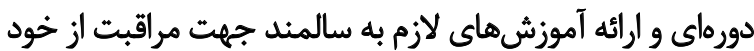

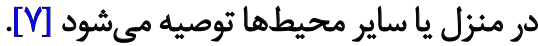

سالمند در كشورهاى در حال توسعه زندكى خواهند كرد. از اين

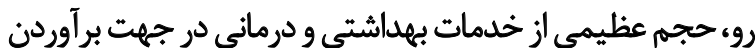

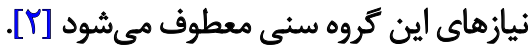
بر اساس تزارش سازمان بهداشت جهانى، ايران در سالهاى

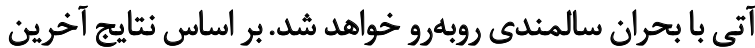

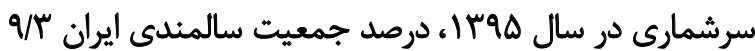

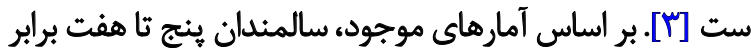

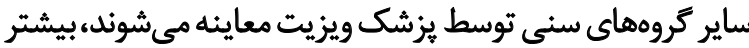

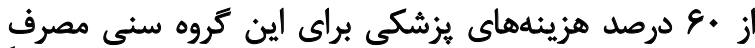

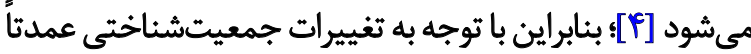

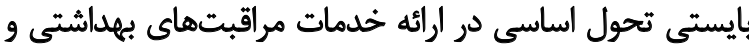

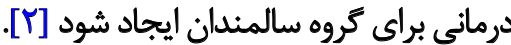
ميزان شيوع بيماريهاى مزمن، ناتوانىهاى جسمانى،

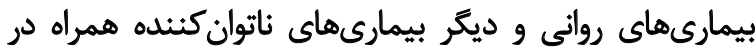

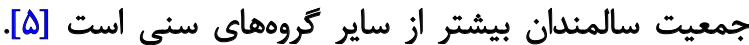

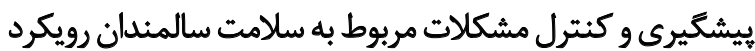

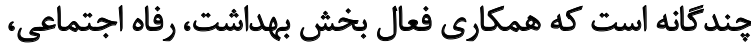

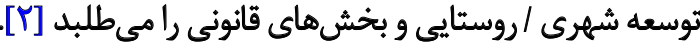
بنابراين لازم است كه برنامه مراقبتهاي بهداشتى سالمندان

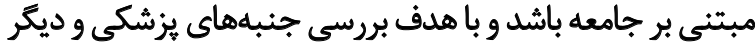

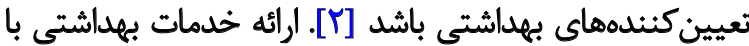

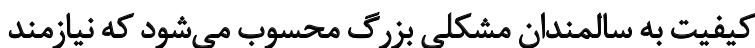

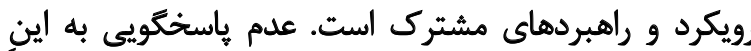

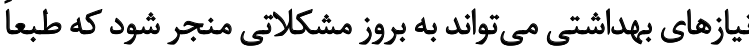

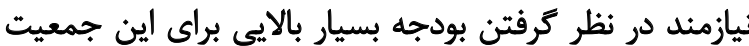

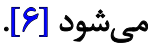

در كشور ايران برنامه ارائه خدمات و مراقبتهاى ادغاميافته در

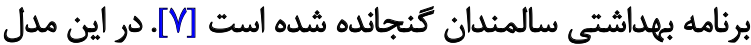

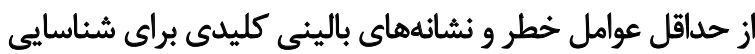

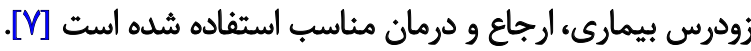

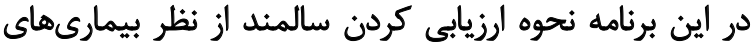

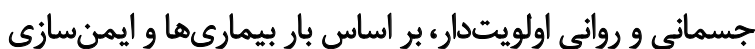

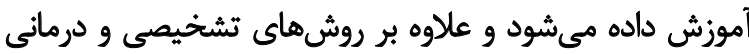
ساده، راههاى يُيشكيرى از بيمارى نيز عنوان شده است.

برنامه جامع مراقبت از سالمند به صورت يك برنامه با عنوان

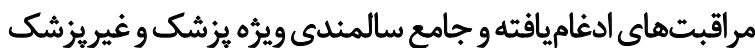

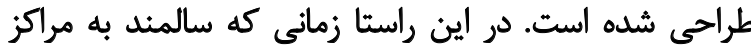

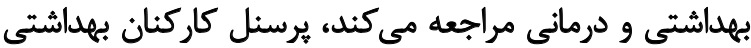

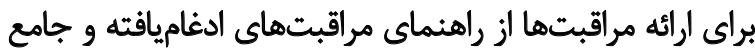

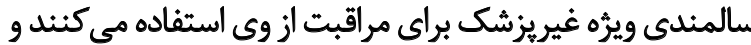

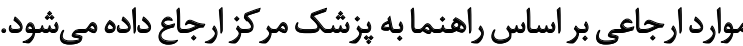
در يايان ارائه مراقبتها جنانجه براي سالمند احتمال ابتلا به 


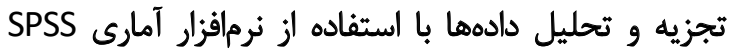

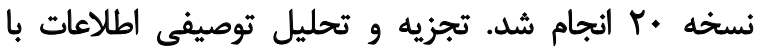

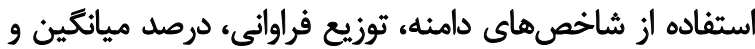

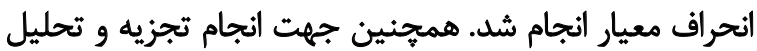

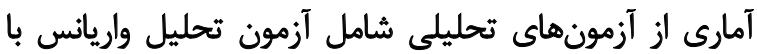

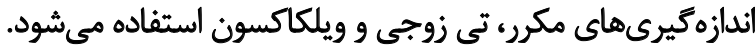

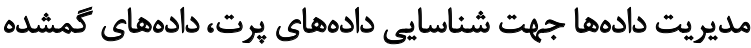
و تعيين نرماليتى انجام شد.

ياقتثلها

در اين مطالعه يرونده الكترونيك سلامت يانصد نفر از سالمندان

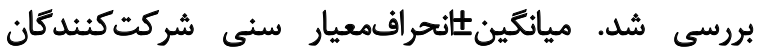
\&q/9VIV/fD

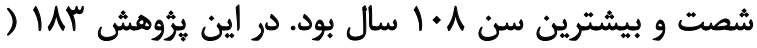

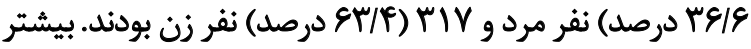

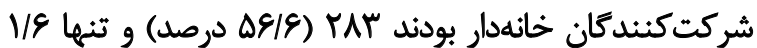

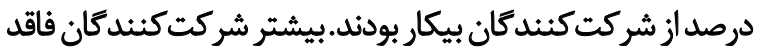

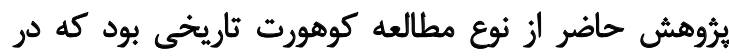

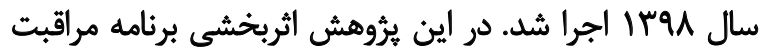

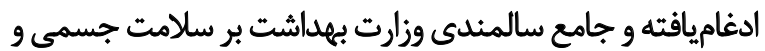

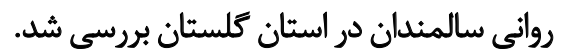
در اين مطالعه برونده الكترونيكى بانصد نفر از سالمندان به

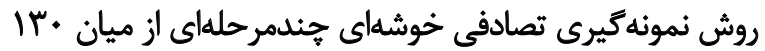

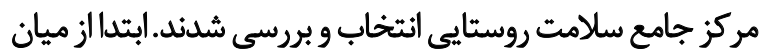

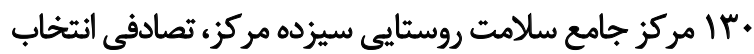

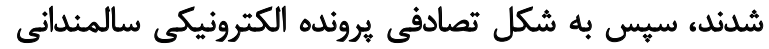

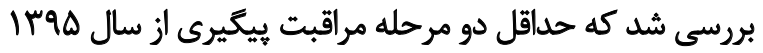

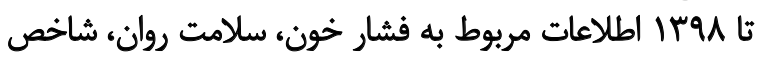

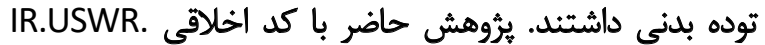
REC.1397.109 توسط دانشعاه علوم توانبخشى و سلامت بافت اجتماعى تهران تصويب شد.

جدول ا. متغيرهاى جمعيتشناختى مربوط به يرونده سلامت سالمندان در مطالعه اثربخشى برنامه مراقبت ادغاميافته و جامع سالمندان

\begin{tabular}{|c|c|c|}
\hline ثعداد (درصد) & \multicolumn{2}{|c|}{ متغير } \\
\hline EN(IH/S) & كشاورز & \multirow{5}{*}{ ت كغل } \\
\hline $\operatorname{rAT}(\Delta \& \mid \varnothing)$ & خانهدار & \\
\hline$e V(1 m / 4)$ & كاركر & \\
\hline$V f(\mid f / \lambda)$ & بازئشسته & \\
\hline$A(1 / 9)$ & بيكار & \\
\hline$r v \cdot\left(M^{q}\right)$ & فاقد تحصيالات رسمى & \multirow{4}{*}{ تحصيلات } \\
\hline$\| \varepsilon(\pi / T)$ & ابتدايى & \\
\hline$g(1 / T)$ & راهنمايى & \\
\hline$A(1 / 8)$ & دييلم & \\
\hline refe(r/A) & مثتاهل & \multirow{3}{*}{ وضعيت تأهل } \\
\hline $9(1 / 1)$ & هركز ازازدواج نكرده & \\
\hline $\operatorname{IrV}(T \Delta / F)$ & هجرد & \\
\hline $1 E 9(\mu f / \Lambda)$ & فارس & \multirow{6}{*}{ قوميت } \\
\hline $9 \Delta(19 / \Delta)$ & تركمن & \\
\hline$M(N A)$ & ترى & \\
\hline $\operatorname{lFa}(\mathrm{rq} / \mathrm{A})$ & سيستانى & \\
\hline$f(\cdot / \Lambda)$ & كرد & \\
\hline$r \cdot(g / \pi)$ & بلوج & \\
\hline
\end{tabular}

证 
جدول r. وضعيت ميزان ثبت شاخصهاى سلامت

\begin{tabular}{|c|c|c|c|c|}
\hline \multicolumn{3}{|c|}{ فراواتى (دروصد) } & \multirow{2}{*}{ وضعيت } & \multirow{2}{*}{ شاخحص } \\
\hline مرحله سوم & مر حله دوم & مرحله أول & & \\
\hline PqI(NNY) & $M(P / P)$ & $r \cdot(8)$ & ثبت نشده & \multirow{2}{*}{ فشار خون سيستوليك } \\
\hline $1.9(r / 1 / 1)$ & $\operatorname{rgn}(q \pi / 9)$ & $P V+(q 4)$ & ثبت شده & \\
\hline एqI(VNT) & $M(\& / F)$ & $r \cdot(\varepsilon)$ & ثبت نشده & \multirow{2}{*}{ فشار خون دياستوليك } \\
\hline $1.9(r / / A)$ & FEN(qT/9) & $P V+(q 4)$ & ثبت شده & \\
\hline $\operatorname{Peq}(q r / A)$ & $r q \Delta(v q)$ & "rqu(NNE) & ثبت نشده & \multirow{2}{*}{ وزن مرحله } \\
\hline$r(5 q / r)$ & $1+\Delta(r))$ & $1 \cdot V(r / / F)$ & ثبت شده & \\
\hline req(qu/A) & $r q .(v 1)$ & พqT(NNP) & ثبت نشده & \multirow{2}{*}{ قد مرحله } \\
\hline$r(g / r)$ & $11+(T Y)$ & $1 \cdot A(r Y / R)$ & ثبت شده & \\
\hline$p q \Delta(q q)$ & $r \Delta(N \cdot / r)$ & $r \Delta \cdot(r \cdot)$ & ثبت نشده & \multirow{2}{*}{ افسرد5ى } \\
\hline$\Delta(Y / *)$ & $\mid F q(r q / A)$ & $10 \cdot(\mu \cdot)$ & ثبت شلده & \\
\hline
\end{tabular}

ill

درصد و در مرحله سوم ا درصد بود. جدول شماره ب ميزان ثبت شاخصهاى سلامت رانشان مي دهد. درصد

جهت ارزيابى فشار خون سيستوليك در سه مرحله تعداد افراد

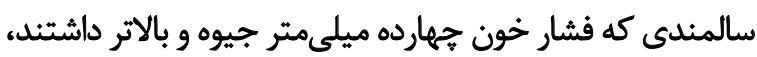

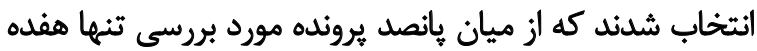

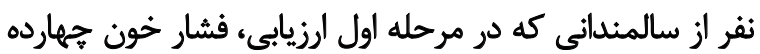

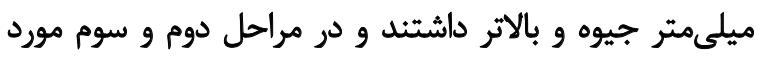
ارزيابى قرار ترفته بودئد، وارد تحليل شدئل

با توجه به بررسى آزمون كرويت ماخلى (آزمون يكنواختى

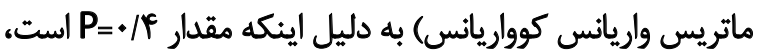

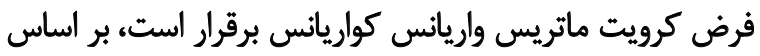

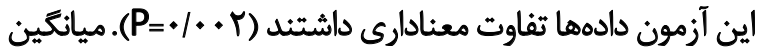

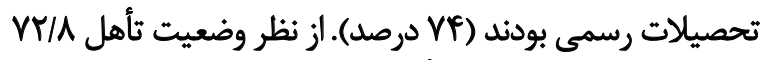

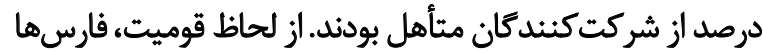

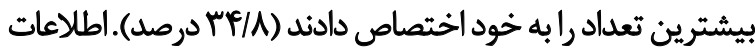

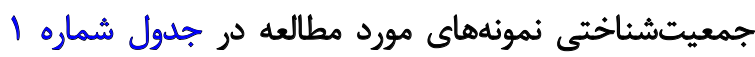

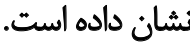

در اين يرؤهش سه متغير شامل افسردمى (يرسشنامه

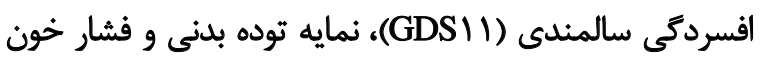

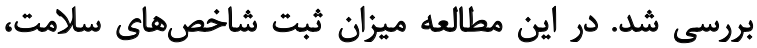

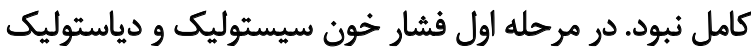

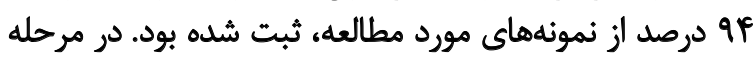

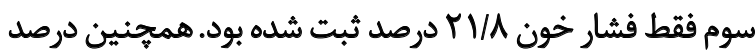

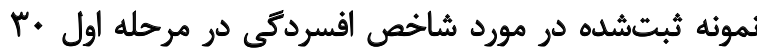

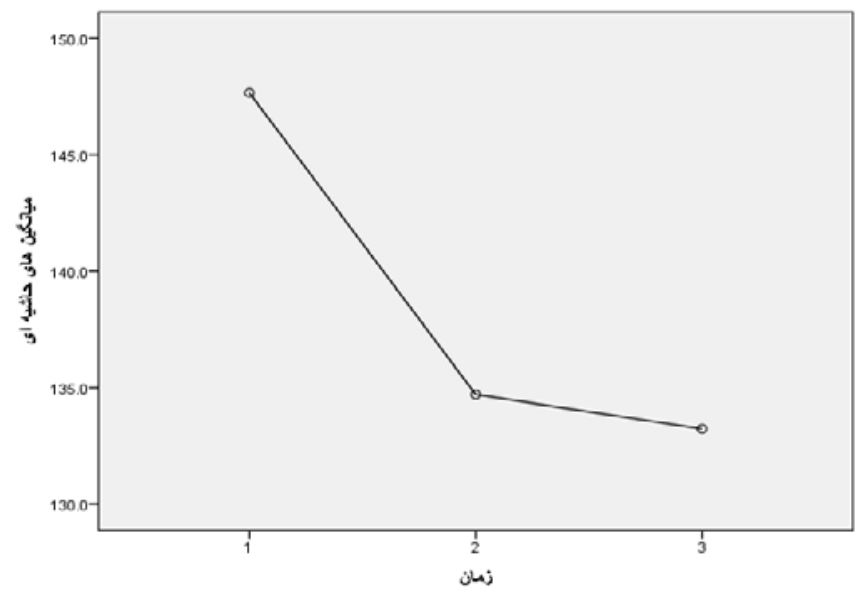


جدول ب. نتايج آزمون تحليل واريانس با ائدازهكيرىهاى مكرر در ميزان فشار خون سيستوليك

\begin{tabular}{|c|c|c|c|c|}
\hline سيح معنادارى & 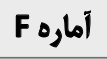 & ميانكينين مربعات & ورجه آزادى & تثيجه آزمون \\
\hline $.1 \cdot . r$ & V/er & 1.89 & r & نتيجه آزمون تحليل واريائس با اثلدازمكيرى هكرر \\
\hline
\end{tabular}

جدول F. نتايج آزمون تحليل واريانس با اندازهكيرىهاى مكرر در ميزان فشار خون دياستوليك

\begin{tabular}{|c|c|c|c|c|}
\hline سطح معنادارى & F آماره F & ميانغَين مربعات & درجه أزادى & نتيجه أزمون \\
\hline.$/ 19$. & $1 / V$ & $11 \% / 14$ & $V / \& \Delta$ & واريانس با اثندازميرى مكرر \\
\hline
\end{tabular}
ن

جدول هـ آزمون تى زوجى در مورد توده بلدى در مراحل اول و دوم

\begin{tabular}{|c|c|c|c|c|c|}
\hline \multicolumn{2}{|c|}{ فاصله اطمينان •و درصد } & \multirow{2}{*}{ سطح معنادارى } & \multirow{2}{*}{ درجه آزادى } & \multirow{2}{*}{ تى - ت } & \multirow{2}{*}{ هتغير } \\
\hline كران بالا & كران ثايين & & & & \\
\hline$+1+4$ & $-+1++\infty$ & $+/ 9+r$ & $1+r$ & $-t / N r$ & توده بلدنى سال هاى اول و دوم \\
\hline
\end{tabular}

il

بررسى آزمون كرويت ماخلحى (آزمون يكنواختى ماتريس واريانس

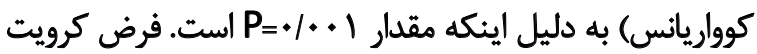

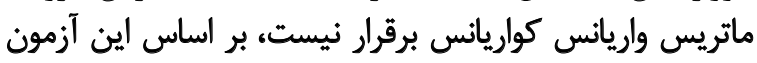

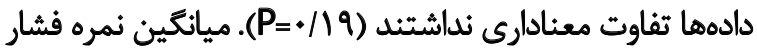

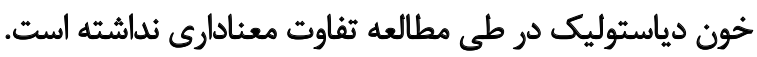

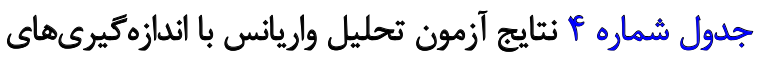

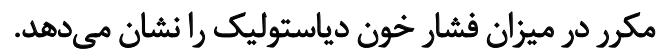

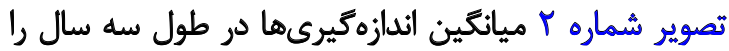

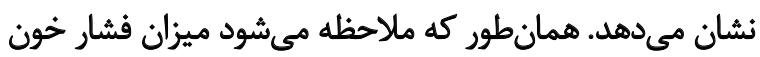

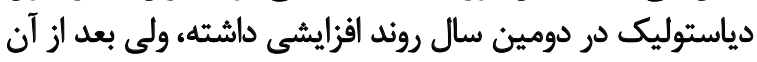

نمره فشار خون در طى مطالعه تفاوت معنادارى داثشئه است.

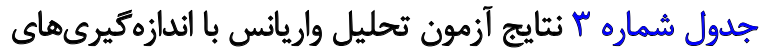
مكرر در ميزان فشار خون سيستوليك ران انشان مى خدهد.

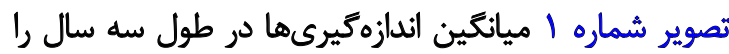

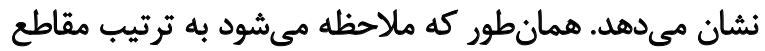

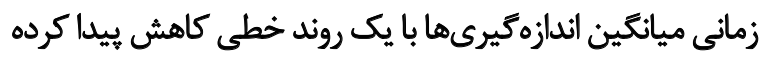
أسث اطلاعات مربوط به فشار خون دياستوليك 9 • ا نفر از سالمندان

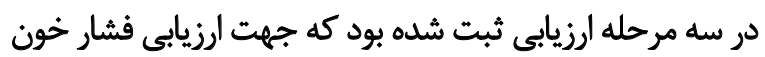

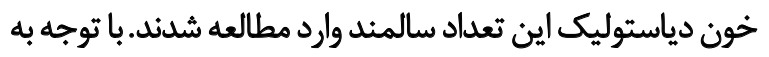

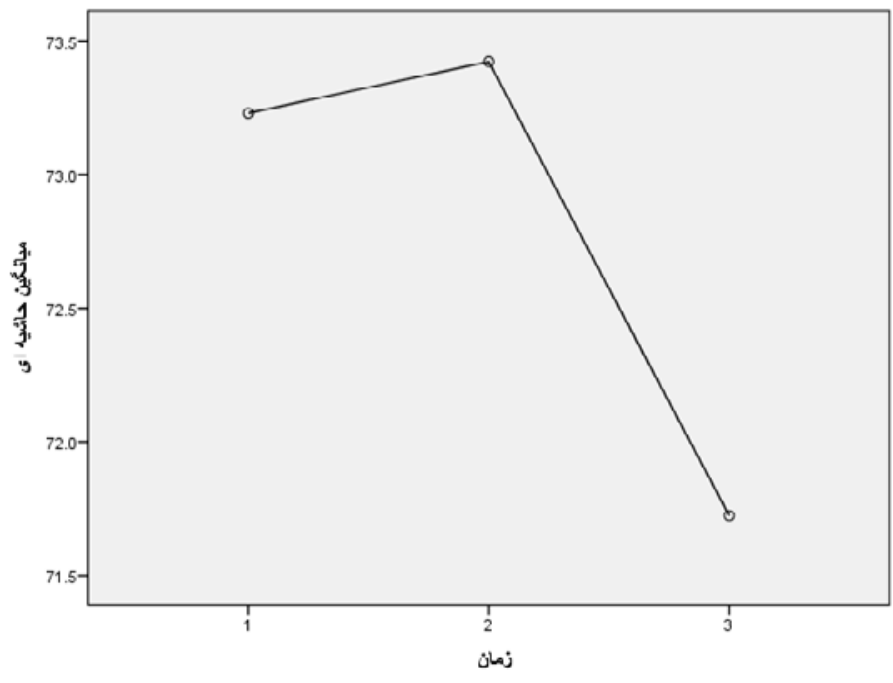


جدول و. طبقهبندى نمره افسردكى در مرحله اول و دوم

\begin{tabular}{|c|c|c|c|c|}
\hline \multicolumn{4}{|c|}{ تعداد (درصد) } & \multirow{2}{*}{ متغير } \\
\hline مر هله دوم & مر حله اول & مر حله دوم & موحله اول & \\
\hline \multicolumn{2}{|c|}{ qfN (M/P) } & \multicolumn{2}{|c|}{ IFY (IHY) } & علدم ابتلا به افسردمى \\
\hline \multicolumn{2}{|c|}{$\Delta / r(11 / / \varphi)$} & \multicolumn{2}{|c|}{$\Lambda(I V)$} & احتمال ابتلال به افسردكى \\
\hline
\end{tabular}

证

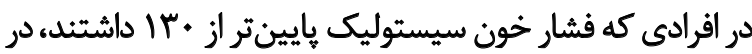

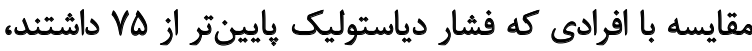

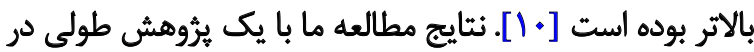

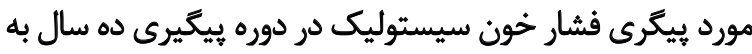

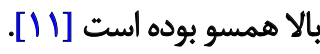

از آنجا كه فشار خون به ازاى افزايش سن در دوران سالمندى

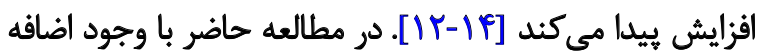

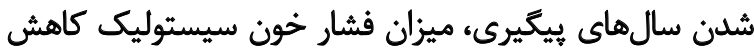

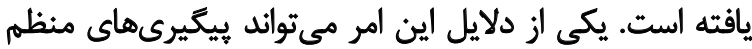

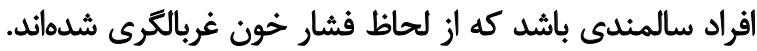

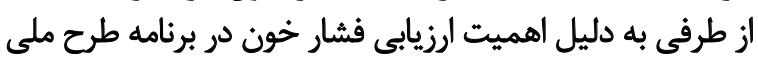

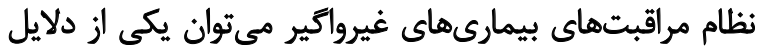

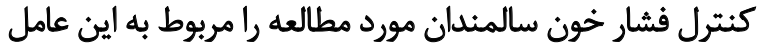

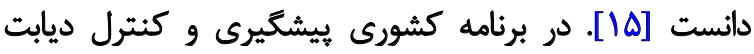

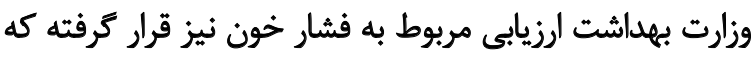

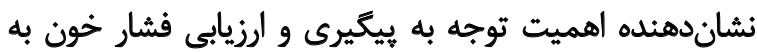

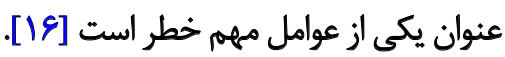

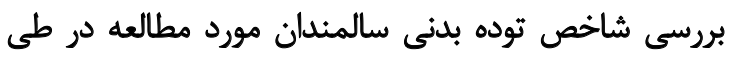

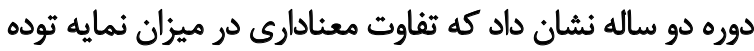

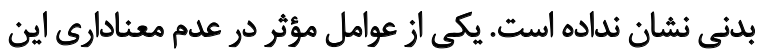

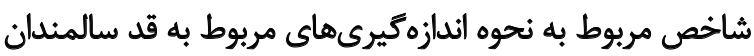

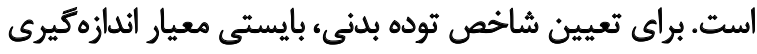

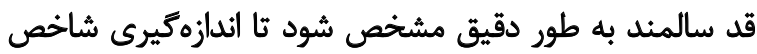

توده بدنى بهدرستى انجام تيرد [IV]

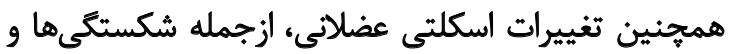

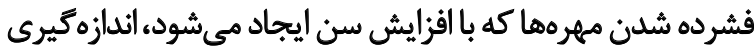

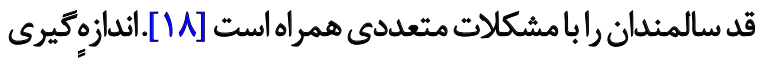

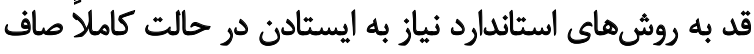

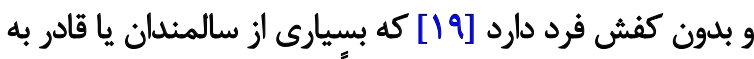

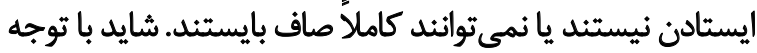

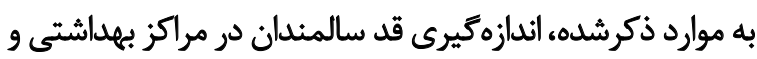

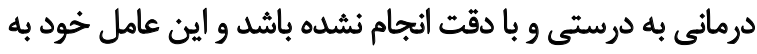

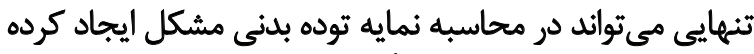

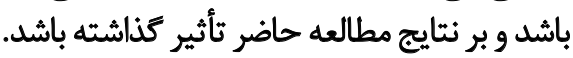

همجنين مطالعه مشابهاى در ايران در زمينه بررسى دورهاي
ميزان فشار خون دياستوليك بعد از سال سوم با يك روند خطى

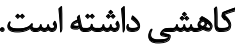

جهت ارزيابى شاخص توده بدنى اطلاعات قد و وزن أن أنفر

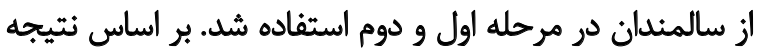

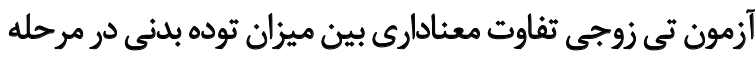

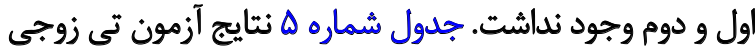
درباره توده بدنى رانشان ميى دهد.

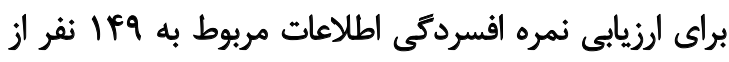

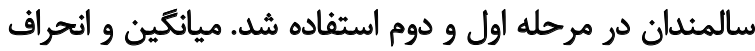

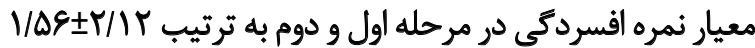

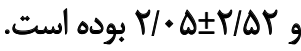

جدول شماره \& نمره افسردگى در سال اول و دوم را بر اساس

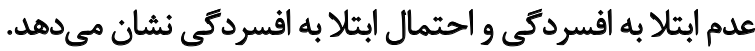

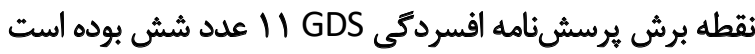

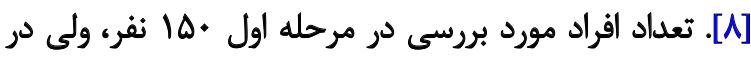

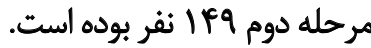

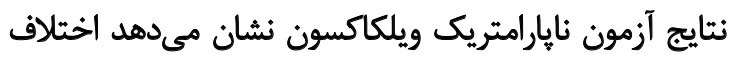

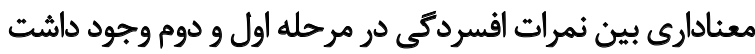
( $P=\cdot / \cdot r)$

$\leftrightarrow$

مطالعه حاضر به منظور تعيين اثربخشى برنامه مراقبت

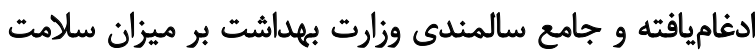

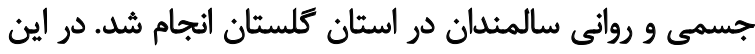

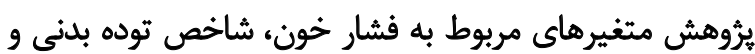

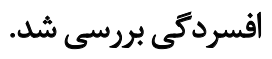

نتايج مربوط به بررسى فشار خون سيستوليك نشان داد كه

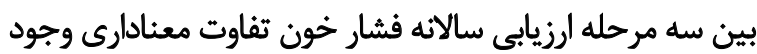

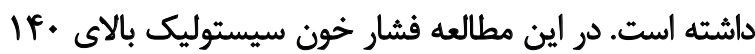

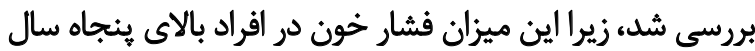

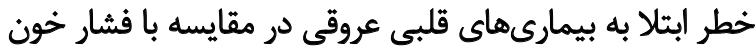

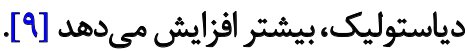
باين حال،در مطالعه كوهورت استكهلم خطر نسبى مركومير 
نمايه توده بلنى اطلاعات آمارى مربوط به دو مرحله اول مناسب

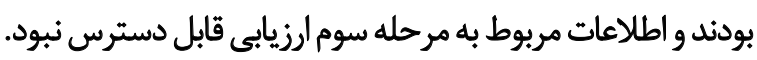
نتايج مطالعه حاضر نشان مي دهد كه برنامه مراقبت ادغاميافته

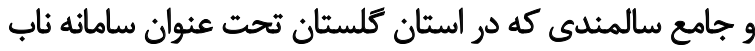

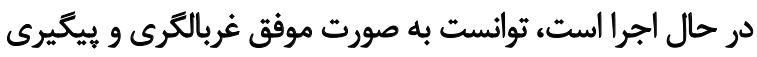

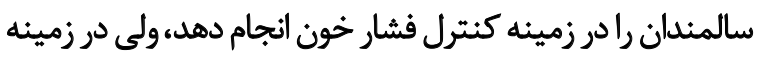

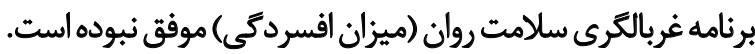

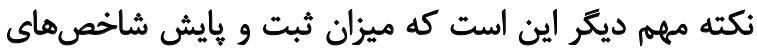
سلامت در حد مطلوب نبوده است.

يبيشنهاد ميشود كه مطالعات آينده به صورت كوهورت آيندهنكر انجام شود و اطلاعات مورد نياز با همكارى بهات بهورز يا يات

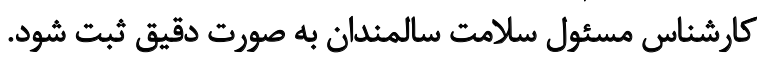

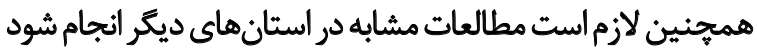
و نتايج مطالعات با يكديخر مقايسه شود، زيرا با توجهات بهات به شرايط

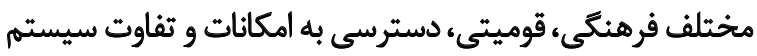

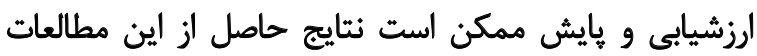

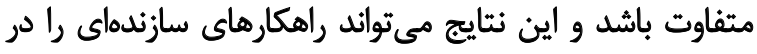

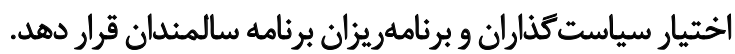

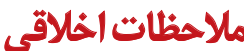 \\ ييروى از اصول الخالاق يُوهش}

IR.USWR.REC.1397.109 توروهش حاضر با كد اخلاقي تواني توسط دانشكاه علوم توانبخشى و سلامت اجتماعى تهران تصويب

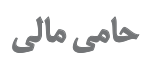

اين تحقيق كرنتث بثرهشى را از مركز تحقيقات سالمندى دانشعاه علوم توانبخشى و سلامث اجتماعى تهرئ تران دريافت كرد.

$$
\text { مشاركت ثويسندكًان }
$$

همه نويسندكان در طراحى، اجرا و نكارش همه بخشهاي

$$
\text { يُروهش مشاركت داشتيند. }
$$

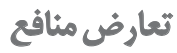

بنابر اظهار نويسندكان اين مقاله تضاد منافع ندارد.

$$
\text { تشيكر و قعدراني }
$$

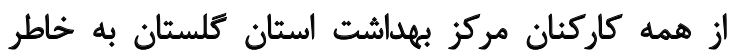

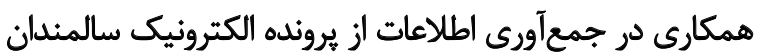
كمال تشكر را داريم.
شاخص توده بدنى در سالمندان انجام نشده و با توجه به اهميت باني

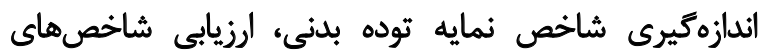

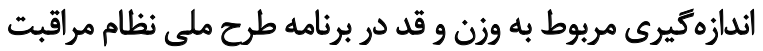

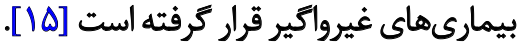
نتايج حاصل از بررسى نمره افسردگى سالمندان مورد مطالعه

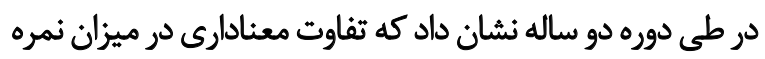

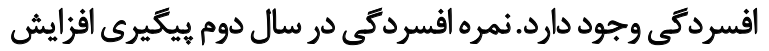

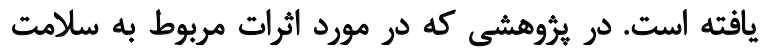

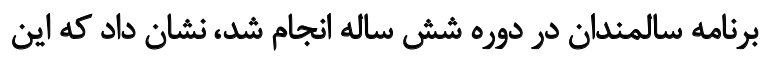

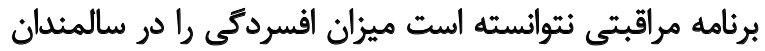

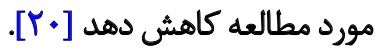

نتايج مطالعه كوهورت در مورد آيندهنكرى عوامل خطر ابتلابه

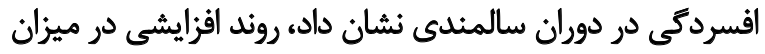

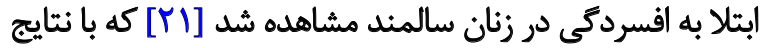

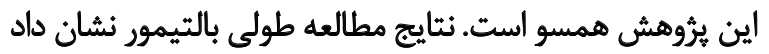

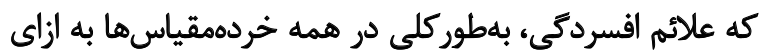

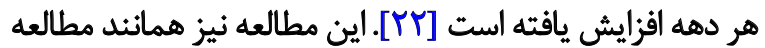

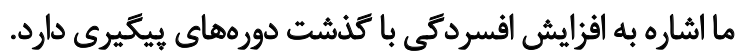

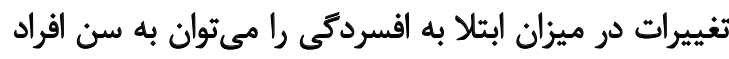

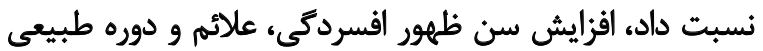

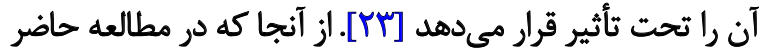

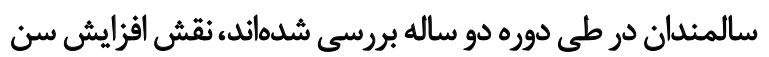
در ميزان افسردكى سالمندان برجستهتر شده است.

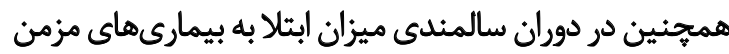

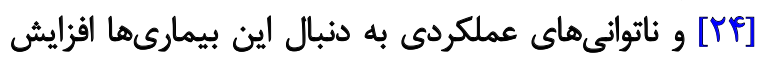

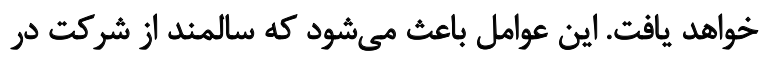

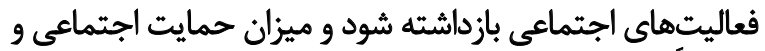

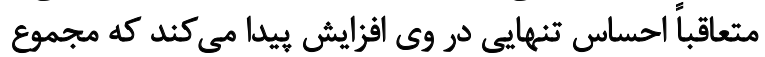

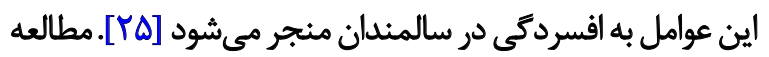

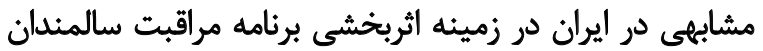
انججام نشيده استيت.

\section{نتيجهَيَيرىنهايي}

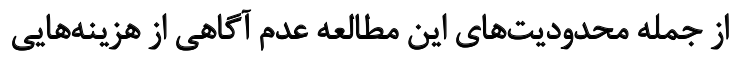

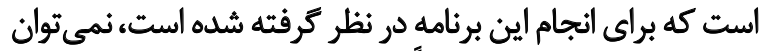

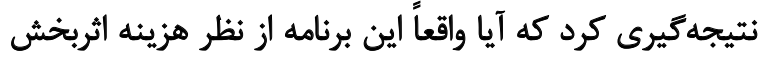

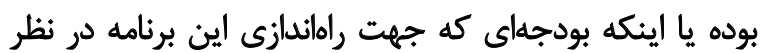

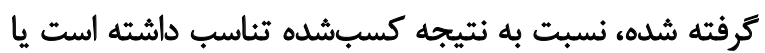

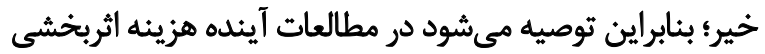
نيز بررسى شود.

اطلاعات مربوط به ثبت شاخصهاي بهداشتى در سه مرحله

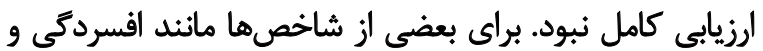




\section{Refrences}

[1] World Health Organization. Definition of an older or elderly person 2013 [Internet]. 2013 [Updated 2015 June 30]. Available from: Link Not Found

[2] World Health Organization. Towards policy for health and ageing [Internet]. 2013 [Updated 2013 May 22]. Available from: http://www.who.int/ageing/publications/alc_fs_ageing_policy.pdf

[3] Statistical Centre of Iran. The statistical yearbook of Iran 2016 [Internet]. 2016 [Updated 2018 April]. Available from: https:/ /irandataportal.syr.edu/wp-content/uploads/Statistical-Yearbook2016-2017-1395-\%E2\%80\%93-Persian-.pdf

[4] Sadoughi F, Shahi M, Ahmadi M, Davaridolatabadi N. Health information management system for elderly health sector: A qualitative study in Iran. Iranian Red Crescent Medical Journal. 2016; 18(2):e21520. [PMID] [PMCID]

[5] Boutayeb A, Boutayeb S. The burden of non communicable diseases in developing countries. International Journal for Equity in Health. 2005; 4:2. [DOI:10.1186/1475-9276-4-2] [PMID] [PMCID]

[6] Shrivastava SRBL, Shrivastava PS, Ramasamy J. Health-care of Elderly: Determinants, needs and services. International Journal of Preventive Medicine. 2013; 4(10):1224-5. [PMID] [PMCID]

[7] Shati M, Azimi MS, Davari S, Solbi Z, Ladoni Sh, Kargozar E, et al. Integrated and comperhensive health care system for elderly people (for health care staff). Tehran: Ministry of Health and Medical Education; 2019. https://arsanjan.sums.ac.ir/Dorsapax/Data/Sub_142/File/salmandan.pdf

[8] Malakouti SK, Fatollahi P, Mirabzadeh A, Salavati M, Zandi T. Reliability, validity and factor structure of the GDS_15 in Iranian elderly. International Journal of Geriatric Psychiatry. 2006; 21(6):588-93. [DOI:10.1002/gps.1533] [PMID]

[9] Chobanian AV, Bakris GL, Black HR, Cushman WC, Green LA Izzo Jr JL, et al. The seventh report of the joint national committee on prevention, detection, evaluation, and treatment of high blood pressure: The JNC 7 report. JAMA. 2003; 289(19):2560-72. [DOI:10.1001/jama.289.19.2560] [PMID]

[10] Guo Z, Viitanen M, Winblad B. Low blood pressure and fiveyear mortality in a Stockholm cohort of the very old: Possible confounding by cognitive impairment and other factors. American Journal of Public Health. 1997; 87(4):623-8. [DOI:10.2105/ AJPH.87.4.623] [PMID] [PMCID]

[11] Dregan A, Ravindrarajah R, Hazra N, Hamada S, Jackson SHD, Gulliford MC. Longitudinal trends in hypertension management and mortality among octogenarians: Prospective cohort study. Hypertension. 2016; 68(1):97-105. [DOI:10.1161/HYPERTENSIONAHA.116.07246] [PMID] [PMCID]

[12] Cheraghi P, Mihandoost Yeganeh Z, Dosti Irani A, Sangestani M, Cheraghi Z, Khezeli M. [Study on the prevalence of hypertension and its associated factors in the elderly population (Persian)]. Journal of Geriatric Nursing. 2015; 1(3):73-86. http://jgn.medilam.ac.ir/article-1-106-en.html

[13] Hoseini SR, Zabihi A, Bijani A. [Prevalence of cardiovascular risk factors in elderly population in Amirshahr (2007) (Persian)]. Salmand: Iranian Journal of Ageing. 2009; 4(1):46-52. https://salmandj.uswr.ac.ir/article-1-332-en.html

[14] Tugay Aytekin N, Pala K, Irgil E, Akis N, Aytekin H. Distribution of blood pressures in Gemlik District, NorthWest Turkey.
Health \& Social Care in the Community. 2002; 10(5):394-401. [DOI:10.1046/j.1365-2524.2002.00379.x] [PMID]

[15] Khodabakhshi H, Tiyuri A, Yari E, Beheshti D, Sharifzadeh GR. [Prevalence of non-communicable disease risk factors among the elderly of Birjand in 2014 (Persian)]. Salmand: Iranian Journal of Ageing. 2019; 14(1):52-63. [DOI:10.32598/sija.13.10.370]

[16] Alirezaei Shahraki R, Aliakbari Kamrani A, Sahaf R, Abolfath Momtaz Y. [Effects of nationwide program for prevention and control of diabetes initiated by the Ministry of Health on elderly diabetic patients' knowledge, attitude and practice in Isfahan (Persian)]. Salmand: Iranian Journal of Ageing. 2019; 14(1):84-95. [DOI:10.32598/SIJA.14.1.84]

[17] Gunnell D, Berney L, Holland P, Maynard M, Blane D, Frankel $\mathrm{S}$, et al. How accurately are height, weight and leg length reported by the elderly, and how closely are they related to measurements recorded in childhood? International Journal of Epidemiology. 2000; 29(3):456-64. [DOI:10.1093/ije/29.3.456] [PMID]

[18] Hickson M, Frost G. A comparison of three methods for estimating height in the acutely ill elderly population. Journal of Human Nutrition and Dietetics. 2003; 16(1):13-20. [DOI:10.1046/ j.1365-277X.2003.00416.x] [PMID]

[19] Nazari N, Fakhrzadeh H, Sharifi F, Arzaghi M, Alizadeh M, Mehrdad N, et al. [Prediction of Iranian aged people height from age, shin length and forearm length (Persian)]. Iranian Journal of Diabetes and Metabolism. 2014; 13(1):1-8. https://ijdld.tums. ac.ir/article-1-5084-en.html

[20] Kim YE, Hong SW. Health-related effects of the elderly care program. BioMed Research International. 2018; 2018:7121037. [DOI:10.1155/2018/7121037] [PMID] [PMCID]

[21] Chang SC, Pan A, Kawachi I, Okereke OI. Risk factors for late-life depression: A prospective cohort study among older women. Preventive Medicine. 2016; 91:144-51. [DOI:10.1016/j. ypmed.2016.08.014] [PMID] [PMCID]

[22] Davey A, Halverson Jr CF, Zonderman AB, Costa Jr PT Change in depressive symptoms in the baltimore longitudinal study of aging. The Journals of Gerontology. Series B, Psychological Sciences and Social Sciences. 2004; 59(6):P270-7. [DOI:10.1093/ geronb/59.6.P270] [PMID]

[23] Bastami F, Salahshoori A,Shirani F, Mohtashami A,Sharafkhan N. [Risk factors of depression on the elderly: A review study (Persian)]. Journal of Gerontology. 2016; 1(2):54-65. [DOI:10.18869/ acadpub.joge.1.2.54

[24] Kim O, Byeon YS, Kim JH, Endo E, Akahoshi M, Ogasawara $\mathrm{H}$. Loneliness, depression and health status of the institutionalized elderly in Korea and Japan. Asian Nursing Research. 2009; 3(2):63-70. [DOI:10.1016/S1976-1317(09)60017-7] [PMID]

[25] Wan Mohd Azam WMY, Din NC, Ahmad M, Ghazali SE Ibrahim N, Said Z, et al. Loneliness and depression among the elderly in an agricultural settlement: Mediating effects of social support. Asia-Pacific Psychiatry. 2013; 5(S1):134-9. [DOI:10.1111/ appy.12061] [PMID] 
This Page Intentionally Left Blank 